\title{
Sprint Fidelis implantable cardioverter- -defibrillators lead patient management and survival: Single center study
}

\author{
Marcin Grabowski ${ }^{1}$, Jakub K. Rokicki ${ }^{1}$, Sylwia Gajda ${ }^{1}$, Łukasz Januszkiewicz ${ }^{1}$, \\ Andrzej Cacko ${ }^{1,2}$, Przemysław Stolarz ${ }^{1}$, Grzegorz Opolski ${ }^{1}$
}

${ }^{1}$ First Chair and Department of Cardiology, Medical University of Warsaw, Poland

${ }^{2}$ Department of Medical Informatics and Telemedicine, Medical University of Warsaw, Poland

\begin{abstract}
Background: Over the last several years significant rises in the use of implanted cardioverter-defibrillators (ICD) have also resulted in a number of associated complications. This number includes lead failure. Sprint Fidelis (SF) ICD lead is regarded as a lead with elevated failure risk. Every center acting in accordance with the guidelines should observe patients more thoroughly especially with recalled leads and run a registry of their follow-up. The aim of this research was to present follow-up of the patients with SF leads (types 6948, 6949) from a single implantation center.

Methods: There were 36 SF leads implanted in 36 patients. Mean follow-up period was 76 months (IQR 40.3-86.8). Patients were subjected to regular check-ups in 3 to 6 month intervals.

Results: Patients were implanted at a median age of 66.5 years and majority of them had ischemic cardiomyopathy (72\%). A majority of the studied population were men (72.2\%). Predominantly dual-chamber ICD (ICD-DR) were implanted (50\% ICD-DR vs. $47.2 \%$ ICD-VR). The guidelines for management of patients implanted with $S F$ were fully implemented. During the follow-up 14 (38.9\%) patients died. No deaths were noted that could be attributed to lead failure. In 5 cases lead failure was identified and of these 4 leads were replaced. Median time from implantation to the detection of lead dysfunction was 52 months (IQR 49; 83). The symptoms of failure consisted of: inappropriate shocks, alternating ventricular lead signal, or loss of ventricular stimulation.

Conclusions: The follow-up of patients with recalled SF leads in a single center supports that implementation SF management guidelines could be effective in clinical practice. (Cardiol J 2017; 24, 3: 259-265)
\end{abstract}

Key words: defibrillators, implantable (E07.305.250.159.175) lead failure, Sprint Fidelis, lead extraction, lead survival

\section{Introduction}

The Sprint Fidelis (SF) 6948 and 6949 models (Medtronic, Minneapolis, Minnesota, USA) are 6.6-French defibrillation leads with bipolar coil, coated with silicone, including a polyurethane overlay and of passive (6948; tines) or active (6949; active screw-in) fixation. This lead family has been in distribution since 2004. The first signals of elevated risk of fracture emerged in July 2007 when Hauser et al. [1] published their study results. In October 2007 Medtronic Inc. issued Urgent Medical Device Information [2] to all physicians and hospitals who were using SF leads stating that "no more SF leads will be sold or manufactured and any remaining product should be pulled from

Address for correspondence: Marcin Grabowski, MD, PhD, FESC, First Chair and Department of Cardiology, Medical University of Warsaw, ul. Banacha 1a, 02-097 Warszawa, Poland, tel: +48 22599 19 58, fax: +48 2259919 57, e-mail: marcin.grabowski@wum.edu.pl

Received: 22.10.2016 Accepted: 26.10.2016 
inventory and returned to the company". Since then, a number of publications have reported a variety of risk factors which may be associated with Fidelis lead fracture. Polish group of experts lead by prof. Adnrzej Kutarski, MD, PhD published in 2011 in 'Kardiologia Polska' journal guidelines for management of patients implanted with SF leads [3]. This document emphasized the importance of consecutive (once every 3 months) control visits to centers of cardiac implantable electronic devices control. According to this document a malfunctioning lead should be replaced with no hesitation. The guidelines also advised prophylactic (elective) replacement of well-functioning lead in three situations: (1) after diagnosing pacing dependency (idiopathic rhythm $<30 / \mathrm{min}$, asystole $>6 \mathrm{~s}$ ); (2) when estimated lifespan is longer than 10 years; (3) on patient's explicit request after understanding the risks and benefits of the extraction procedure. Proper identification of each patient's risk factors and well-managed regular control visits are key to perpetuate proper functioning of implanted devices and reduce the risk of sudden cardiac death, if a lead defect has been identified [4].

The decision to publish experiences with SF leads has been undertaken for the following three reasons: (1) to provide information about failure rate of 6948 and 6949 SF leads in a single center, (2) redefine and assess the importance of risks factors according to a population implanted in the studied hospital, and (3) make findings available for informed management of patients with malfunctioning leads.

\section{Methods}

\section{Patients follow-up}

We enrolled all the patients implanted with SF leads at First Chair and Department of Cardiology, Medical University of Warsaw, Poland. The follow-up visits were initially planned according to manufacturer guidelines annually at this Outpatient Clinic. When Medtronic Inc. (Minneapolis, Minnesota, USA) published a report on increased risk of lead failure [2] an interrogation interval had been scheduled for 3-6 months (according to patient status severity). In accordance with guidelines issued by Kutarski et al. [3], patients were recalled to the preferred contact scheme. When the preferred contact scheme had failed in establishing any communication with the patient or a relative, a registered letter had been sent to the last known postal address. After collecting the data for the entire group, we received information on the status of each person implanted with SF lead (living/deceased and date of death) from the PESEL Database (Universal Electronic System for Registration of the Population) managed at the time of the research by the Ministry of Administration and Digitization of Poland. We had also obtained information on each patient's status before implantation from three sources: IMPULS System provided by Institute of Medical Technology and Equipment, Zabrze, Poland used for registration of patients with cardiac implantable electronic devices (CIED), where patient's data is stored by the clinic's staff members, protocols from procedures and data from the hospital discharge cards. Clinical and device information gathering results were obtained from our ICD database. Additional data was retrieved from clinical records. General demographic data was also collected.

We acknowledged any death that can be assessed as lead-dependent and every sudden cardiac death was reported by the PESEL system. Deaths were described as cardiac, non-cardiac and unknown, according to their cause [5], and the status of "sudden" was attributed, provided the death occurred in less than $1 \mathrm{~h}$ from the beginning of symptom presentation [6].

Lead failure was diagnosed when one or more of the well established criteria [6-9] were fulfilled: (i) a sudden rise in long-term pacing or highvoltage impedance, (ii) electrical noise artefacts as manifested by non-physiological signals on the electrogram or by device diagnostics (e.g. nonphysiological short intervals and/or recurrent nonsustained ventricular tachycardia with intervals usually $<220 \mathrm{~ms}$ ), (iii) failure to sense R-waves or ineffective electrical therapy due to an apparent structural lead defect.

Each patient obtained comprehensive and detailed information about the elevated risk of lead fracture, as well as the risks of transvenous lead extraction procedure. All procedures were performed by an electrophysiologist trained in lead extraction in a hybrid cardiosurgical operating room with a cardiac surgeon on stand-by.

The study assessed patients' survivability, lead failure and associated symptoms. It also described actions in case of an electrode dysfunction. In reference to the leads, the procedure in each case was in accordance with Polish guidelines.

\section{Statistical analysis}

Categorical variables were presented as absolute numbers and percentages. Quantitative variables were expressed as means (standard deviation 
[SD]) and interquartile ranges (IQR). Categorical variables were compared using the Fisher test or $\chi^{2}$ test. Student $t$ test was used to compare continuous variables. Kaplan-Meier analysis was performed to assess survival free from lead failure. Cox regression model was used to establish mortality predictors. Statistical analyses were performed using Statistica software. P value $<0.05$ was considered statistically significant.

\section{Results}

\section{Baseline}

Thirty-six SF leads (17 model 6948, 19 model 6949) were implanted in 36 patients. Baseline patient characteristics is shown in Table 1. Median follow-up time was 76 months (IQR 40.3-86.8). The mean left ventricular ejection fraction (LVEF) was $34.6 \% \pm 13.0$ and only 4 patients had LVEF higher than 50\%. ICD-DR were implanted in half of the patients, ICD-VR in $47.2 \%$ and only $2.8 \%$ were CRT-D. During follow-up the battery was replaced in $18(50 \%)$ patients.

Patients were implanted at median age of 66.5 years (IQR 57.3 and 72 ) and majority of them had etiology of ischemic cardiomyopathy ( $72 \%$ vs. $19 \%$ of dilated cardiomyopathy, $3 \%$ of hypertrophic cardiomyopathy and $6 \%$ classified as other). Other etiology included long QT syndrome (3\%) and myocarditis (3\%). Leads were implanted predominantly through the left cephalic vein access (63.9\%). Among the studied group, all patients received dual-coil leads, 17 (47\%) patients received a 6948 model SF lead (a passive fixation model), and 19 (53\%) a 6949 (an active fixation model). All ICDs were implanted in the prepectoral area. Lead integrity alert was activated in $12(33 \%)$ patients. CareLink system was not used (Table 1).

\section{Lead failure}

A total of 5 lead failures occurred in this cohort. The cumulative overall failure rate for SF leads in our center reached $13.9 \%$, with $23.5 \%$ for the 6948 model, and $5.3 \%$ for the $6949, \mathrm{p}=0.12$. In the group of patients with SF lead failure, the following malfunctions occured: electric noise in 3 patients, and inappropriate shock secondary to electric noise in 2 patients, the low ventricular sensing in 2 patients. We noted ICD interventions (antitachycardia pacing) and shocks in both groups. Overall rate of ICD shocks was 68 (53 in failed $\mathrm{SF}$ group and 15 in the non-failed SF group) with 15 interventions lacking preceding arrhythmic events ( 2 in patients with non-failed leads). ICD interven-
Table 1. Patient characteristics

\begin{tabular}{lc}
\hline Parameter & Overall \\
\hline Follow-up [years], median, IQR & $6.3(3.4-7.2)$ \\
Age at implantation [years] & $66 \pm 11.9$ \\
Men & $26(72.2 \%)$ \\
Women & $10(27.8 \%)$ \\
LVEF [\%], mean \pm SD & $34.6 \pm 13.0$ \\
Etiology: ischemic heart disease & $26(72.2)$ \\
Hypertrophic cardiomyopathy & $1(2.8 \%)$ \\
Dilated cardiomyopathy & $3(8.3 \%)$ \\
Other & $1(2.8 \%)$ \\
Primary prevention & $28(77.8 \%)$ \\
Secondary prevention & $8(22.2 \%)$ \\
Access vein: & \\
Cephalic & $23(63.9 \%)$ \\
Subclavian & $13(36.1)$ \\
Type of device: & \\
ICD-VR & $17(47.2 \%)$ \\
ICD-DR & $18(50 \%)$ \\
CRT-D & $1(2.8 \%)$ \\
Lead tip position: & $11(30.6 \%)$ \\
Right ventricular outflow track & $24(66.7 \%)$ \\
Apex & $17(4.8 \%)$ \\
Other & \\
Active & \\
Passive & \\
\hline & \\
\hline & $19.2 \%)$ \\
\hline
\end{tabular}

IQR - interquartile range; ICD-DR — dual-chamber implantable cardioverter defibrillator; ICD-VR — single-chamber implantable cardioverter defibrillator; CRT-D — implanted cardiac resynchronization defibrillator; IQR - interquartile range; LVEF - left ventricular ejection fraction; SD - standard deviation

tions in the form of antitachycardia pacing were more common in failed-SF group with 148 (of 156 in both group). No significant difference has been observed in the characteristics of patients receiving ICD in primary vs. secondary prevention in received therapies. Median time from implantation to detection of the lead fracture was 52 months (IQR 49 ; 83). Lead failure was diagnozed after $45,49,52$, 66 and 100 months, respectively after implantation in subsequent patients. Lead failure was detected after ICD replacement due to elective replacement indicator in 2 patients ( 0 and 12 months after the replacement procedure, respectively). $80 \%$ of the patients with lead failure underwent a procedure of ICD replacement due to elective replacement indicator. The 1-year, 3-year, 5-year and 7-year lead failure was $0 \%, 0 \%, 9 \%, 33 \%$, respectively (Fig. 1). 


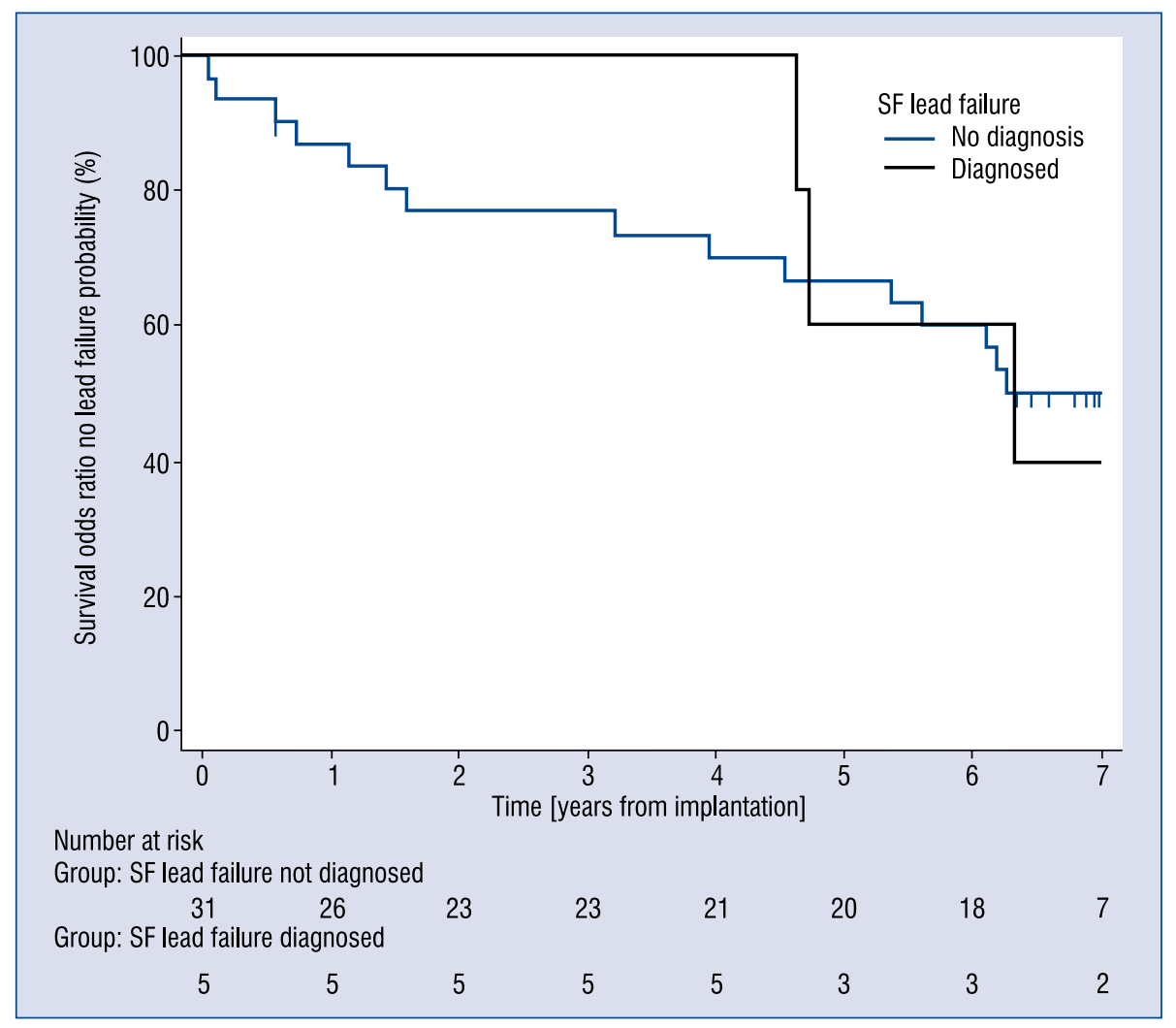

Figure 1. Survival probability in groups with and without the diagnosis of Sprint Fidelis (SF) lead failure.

In Figure 1 we present Kaplan-Meier curves for survival without SF lead failure. The rise of SF lead failure is not associated with the loss of patients from the non-diagnosed group (either deceased or lost to follow-up) in the 7-year follow-up. In this population, patients with dysfunctional lead had lower LVEF (median $26 \%$ vs. $33 \%$, p = 0.34).

\section{Lead management strategies}

Leads were extracted in 6 patients. Four of them were extracted after failure diagnosis. One patient after receiving a complete information on the elevated risk of SF lead failure made a decision about elective replacement, no signs of ICD or lead dysfunction were present. In 1 patient SF lead was extracted because of infective endocarditis. No signs of lead failure were present. No complications were observed during transvenous lead extraction procedures.

\section{Mortality}

During the follow-up 15 (42\%) patients died after a median time from implantation of 38 months (IQR 11.5-69.0). Kaplan-Meier survival probability curves in subgroups of patients with SF lead are presented in Figure 2. Among this group no death can be objectively attributed to an ICD lead failure. In non-survivor groups there were trends towards more frequent implantation in secondary prevention in $(93 \%, \mathrm{n}=14$; vs. $12, \mathrm{p}=0.065)$ and older patients (median 69.0 years old [IQR 63; 76] vs. 66.5 [IQR 57.0; 71.0]; $\mathrm{p}=0.09$ ) than in survivors.

\section{Discussion}

This single-center research provides important data on SF lead recipient outcomes. In particular, we found comparable to other studies' failure rates during long-term follow-up. The cumulative overall failure rate for SF leads in our center reached $13.9 \%$, with $23.5 \%$ for 6948 model, and $5.3 \%$ for 6949 model. Failure rate for only 6948 model was higher than those reported by Medtronic Inc. with the active fixation model rate lower than this provided by the CareLINK Plus (at 8 year survival $7.3 \%$ and $16 \%$, respectively) $[10,11]$. Moreover, it was observed that due to application of the management guidelines fractured SF leads probably did not significantly affect patients' survival during long-term follow-up. 


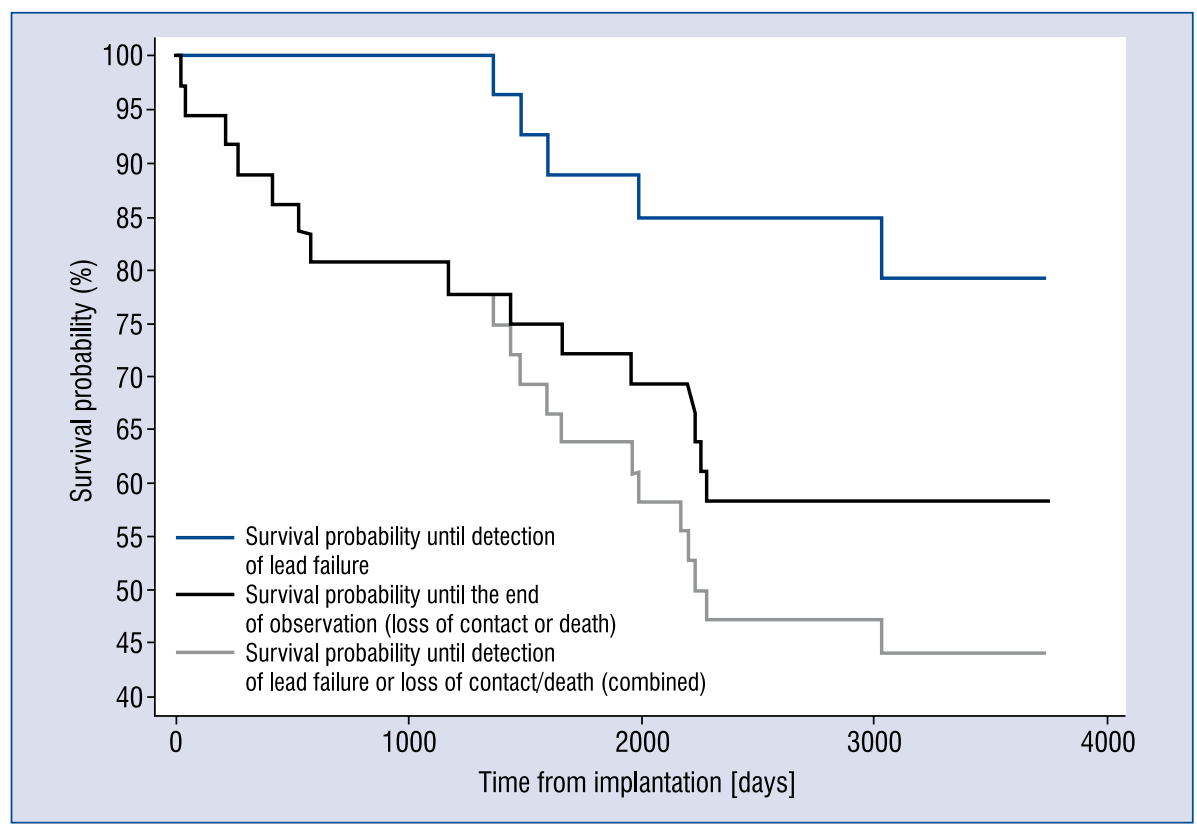

Figure 2. Survival probability in subgroups of patients with implanted Sprint Fidelis (SF) lead.

\section{Lead failure}

Several studies reported elevated lead failure rate of SF leads during long-term follow-up. Most of them presented active fixation lead failure rate which was $9.1-16.4 \%$ in the 5 -year follow-up $[7,12,13]$. By contrast, passive fixation lead failure ranges from $4.2 \%$ to $14.4 \%$ in the 5 -year observation $[6,7,10,14]$.

In present analysis lead failure rate was $9 \%$ at 5 years, which is a relatively low value compared with other literature $[6,7,12,15,16]$. A relatively low lead failure rate was achieved with implantations which involved only double-coil lead in all patients. Tzogias et al. [17] presented similar 9.7\% failure rate in 971 patients with 6949 lead (double-coil) in a 5-year follow-up. Piot et al. [15] found that singlecoil lead had over 3-times higher risk of fracture compared with double-coil lead. Non-cephalic access was used in less than $40 \%$ of the studied population and ICDs were implanted prepectorally in all patients. Both of these factors are claimed to be proteting from lead failure $[7,15,18]$.

During the subpectoral ICD implantation acute bend of the lead is seen often. Moreover, lead might be damaged because of the constantly repeated contraction of the pectoralis muscle [18]. It is not clear why non-cephalic access increases the risk of lead failure. There is a possibility that cephalic access might decrease the hazard of subclavian crush syndrome. Recent reports by Krahn et al. [19] also showed that ICD replacement cannot be attributed to a higher SF lead failure rate This supports the presented findings.

It is known that the lead is the most vulnerable element of ICD system. Therefore, all actions which decrease the risk of lead failure rate should be taken, including cephalic access during implantation. As survival of the patients with ICD and pacemakers increases, so does the risk of complications. Alternative therapies are needed. One of them is subcutaneous ICD, where the lead is located outside of the cardiovascular system [20]. However, the lead is still present in this therapy and it may also have typical lead complications. It follows that lead complications could be avoided only after implementation of various techniques. Fusion of subcutaneous impulse generator and wireless pacemaker would avert the risk of lead failure.

Due to rising interest in establishing predictors of higher risk lead damage [7], it has presently been taken into consideration, gathered demographic and clinical data in order to reveal whether trends were observed elsewhere which may apply to our population of patients was considered as well. This study has not confirmed previous findings [21-24] that patients with confirmed SF lead fracture have statistically significantly higher LVEF, the results obtained herein present contrary results - in our population patients with dysfunctional lead had lower LVEF. Additionally, no statistically significant correlation has been found according to gender 
$[7,9,12,25]$ or age at implantation [23]. Neither was any statistical correlation found between the type of prevention (primary vs. secondary), venous access [7, 18, 21, 24, 26, 27] (cephalic vs. other), nor position of the lead tip in the right ventricle (apex vs. right ventricular outflow track). All parameters were analyzed and were found to be insignificant in multivariate analysis, as well in a univariate analysis.

\section{Management}

Data presented could potentially help physicians implement recommendations with regard to recalled leads. Management of patients with SF lead involves several steps which are recommended. First, outpatient clinic visits are advised to be scheduled more frequently, optimally at 3-month intervals. Second, during information gathering physicians are well advised to look for any signs of lead malfunction. In such cases, the lead could be extracted and a new lead implanted. Third, each patient should be comprehensively informed about the risks associated with the SF lead. Decisions about specific management should be discussed with each patient individually. No incidence was observed where influence of fractured failure lead influenced mortality. Advisory guidelines were applied which might be regarded as contrary to the prophylactic extraction of normally functioning SF lead. A position, first presented in Polish guidelines regarding SF lead management [3] was recently supported by Salgado et al. [28] who reported that in a number of Spanish centers preventive SF had no superior outcome compared to observation and replacement scheduled during generator exchange. This study confirms that the SF lead expert recommendations could be implemented safely and effectively.

\section{Mortality}

No single death could be categorized as associated with lead defect, which concurs with other studies [7, 8, 23, 24, 29]. In the literature, there are slightly lower mortality rates in SF recipients [6]. The trends of this study show quite the same results as in the literature - in non-survivor groups, more frequent implantation was in secondary prevention $[9,23]$ and in older ages [9] than in survivors. ICD interventions without preceding arrhythmic event also do not worsen clinical state of the patients just as Mehta et al. [29] has reported. Up to the time of this report, in United States, Medtronic Inc. associated 13 deaths with SF failure [30].

\section{Limitations of the study}

There are several limitations of the present study. First, the research was based on retrospective analysis of prospectively collected data of limited, single-center cohort. Second, we do not have information on the returned SF leads. Third, causes of death could not be verified post mortem and therefore lead failure rate might be underestimated.

\section{Conclusions}

This study presents the follow-up of patients with recalled SF leads in a single center cohort, which supports the position that implementation of SF management guidelines lead could be effective in clinical practice. The failure rate was $13.89 \%$ which is similar to data reported in other cohorts.

Conflict of interest: Marcin Grabowski declared non-significant $(<10.000 \mathrm{USD})$ role as principal investigator at CareLink Registry, lecturer function and travel support from Medtronic Inc., and serving as a consultant to Sprint Fidelis Polish Expert Group. Andrzej Cacko declared non-significant compensation for participation with a speaker bureau, honoraria for speeches at conferences. Jakub Rokicki, Sylwia Gajda, Łukasz Januszkiewicz, Przemysław Stolarz and Grzegorz Opolski declare no competing interests.

\section{References}

1. Hauser RG, Kallinen LM, Almquist AK, et al. Early failure of a small-diameter high-voltage implantable cardioverter-defibrillator lead. Heart Rhythm. 2007; 4(7): 892-896, doi: 10.1016/j. hrthm.2007.03.041, indexed in Pubmed: 17599673.

2. Medtronic. Urgent Medical Device Information: Sprint Fidelis Lead Patient Management. Recommendations.2007. http:// www.medtronic.com/product-advisories/physician/sprint-fidelis/ PROD-ADV-PHYS-OCT (2007-10-15).

3. Kutarski A, Chudzik M, Mitkowski P, et al. Postępowanie w przypadku pacjentów $z$ elektrodami $z$ rodziny Sprint-Fidelis - wytyczne Zespołu Ekspertów Konsultanta Krajowego w dziedzinie kardiologii. Kardiol Pol. 2011; 69: 995-996.

4. Catanchin A, Anderson L, Jones S, et al. When life-saving devices terminate life. J Cardiovasc Electrophysiol. 2008; 19(3): 316-318, doi: 10.1111/j.1540-8167.2007.01080.x, indexed in Pubmed: 18266677.

5. Epstein AE, Carlson MD, Fogoros RN, et al. Classification of death in antiarrhythmia trials. J Am Coll Cardiol. 1996; 27(2): 433-442, indexed in Pubmed: 8557917.

6. Vollmann D, Woronowicz S, Kmiec L, et al. Passive-fixation lead failure rates and long-term patient mortality in subjects implanted with Sprint Fidelis electrodes. Europace. 2014; 16(2): 258-264, doi: 10.1093/europace/eut185, indexed in Pubmed: 23813451.

7. Birnie DH, Parkash R, Exner DV, et al. Clinical predictors of Fidelis lead failure: report from the Canadian Heart Rhythm 
Society Device Committee. Circulation. 2012; 125(10): 12171225, doi: 10.1161/CIRCULATIONAHA.111.053744, indexed in Pubmed: 22311781.

8. Hauser RG, Maisel WH, Friedman PA, et al. Longevity of Sprint Fidelis implantable cardioverter-defibrillator leads and risk factors for failure: implications for patient management. Circulation. 2011; 123(4): 358-363, doi: 10.1161/CIRCULATIONAHA.110.975219, indexed in Pubmed: 21242478.

9. Morrison TB, Friedman PA, Kallinen LM, et al. Impact of implanted recalled sprint Fidelis lead on patient mortality. J Am Coll Cardiol. 2011; 58(3): 278-283, doi: 10.1016/j.jacc.2011.03.027, indexed in Pubmed: 21737019.

10. Brown J, Bilchick K, Alonso A, et al. OptiVol Impedance Threshold Crossing Predicts Patients with Higher Mortality or Hospitalization Risk Among Medicare Recipients. J Cardiac Failure. 2015; 21(8): S122, doi: 10.1016/j.cardfail.2015.06.350.

11. CareLINK Plus data: Sprint Fidelis model 6949 lead performance. http://wwwmedtroniccom/product-advisories/physician/ sprint-fidelis/6949-LEAD-PERFORMANCE (January 2015).

12. Cheung JW, Tobin-Hess A, Patel A, et al. Trends in Fidelis lead survival: transition from an exponential to linear pattern of lead failure over time. Circ Arrhythm Electrophysiol. 2012; 5(5): 906-912, doi: 10.1161/CIRCEP.112.972000, indexed in Pubmed: 22923271.

13. Parsonnet V, Roelke M. The cephalic vein cutdown versus subclavian puncture for pacemaker/ICD lead implantation. Pacing Clin Electrophysiol. 1999; 22(5): 695-697, indexed in Pubmed: 10353126.

14. Frey SM, Sticherling C, Bucher U, et al. Long-term performance of the Medtronic Sprint Fidelis lead: a matter of lead type? Europace. 2012; 14(11): 1620-1623, doi: 10.1093/europace/eus181, indexed in Pubmed: 22707490.

15. Piot O, Deballon R, Nitu D, et al. Factors predicting Sprint Fidelis lead fracture: Results at 5 years from a French multicentre registry. Arch Cardiovasc Dis. 2015; 108(4): 220-226, doi: 10.1016/j. acvd.2014.11.006, indexed in Pubmed: 25858538.

16. Lovelock JD, Patel A, Mengistu A, et al. Generator exchange is associated with an increased rate of Sprint Fidelis lead failure. Heart Rhythm. 2012; 9(10): 1615-1618, doi: 10.1016/j. hrthm.2012.06.009, indexed in Pubmed: 22683747.

17. Tzogias L, Bellavia D, Sharma S, et al. Natural history of the Sprint Fidelis lead: survival analysis from a large single-center study. J Interv Card Electrophysiol. 2012; 34(1): 37-44, doi: 10.1007/s10840-011-9647-0, indexed in Pubmed: 22314669.

18. Bernstein NE, Karam ET, Aizer A, et al. Right-sided implantation and subpectoral position are predisposing factors for fracture of a 6.6 French ICD lead. Pacing Clin Electrophysiol. 2012; 35(6): 659-664, doi: 10.1111/j.1540-8159.2012.03357.x, indexed in Pubmed: 22469148.
19. Krahn AD, Bashir J, Birnie DH, et al. Impact of generator replacement on the risk of Fidelis lead fracture. Heart Rhythm. 2016; 13(8): 1618-1623, doi: 10.1016/j.hrthm.2016.05.001, indexed in Pubmed: 27154229.

20. Burke MC, Gold MR, Knight BP, et al. Safety and Efficacy of the Totally Subcutaneous Implantable Defibrillator: 2-Year Results From a Pooled Analysis of the IDE Study and EFFORTLESS Registry. J Am Coll Cardiol. 2015; 65(16): 1605-1615, doi: 10.1016/j.jacc.2015.02.047, indexed in Pubmed: 25908064.

21. Ha ACT, Vezi BZ, Keren A, et al. Predictors of fracture risk of a small caliber implantable cardioverter defibrillator lead. Pacing Clin Electrophysiol. 2010; 33(4): 437-443, doi: 10.1111/j.15408159.2009.02626.x, indexed in Pubmed: 19954500.

22. Arias MA, Domínguez-Pérez L, Toquero J, et al. [Sprint fidelis defibrillation lead: a nine-center experience in Spain]. Rev Esp Cardiol. 2011; 64(4): 312-318, doi: 10.1016/j.recesp.2010.11.008, indexed in Pubmed: 21377260.

23. Faulknier BA, Traub DM, Aktas MK, et al. Time-dependent risk of Fidelis lead failure. Am J Cardiol. 2010; 105(1): 95-99, doi: 10.1016/j.amjcard.2009.08.655, indexed in Pubmed: 20102898 .

24. Farwell D, Green MS, Lemery R, et al. Accelerating risk of Fidelis lead fracture. Heart Rhythm. 2008; 5(10): 1375-1379, doi: 10.1016/j.hrthm.2008.06.024, indexed in Pubmed: 18783996.

25. Verlato R, Facchin D, Catanzariti D, et al. Clinical outcomes in patients with implantable cardioverter defibrillators and Sprint Fidelis leads. Heart. 2013; 99(11): 799-804, doi: 10.1136/ heartjnl-2012-303259, indexed in Pubmed: 23434626.

26. Parsonnet V, Roelke M. The cephalic vein cutdown versus subclavian puncture for pacemaker/ICD lead implantation. Pacing Clin Electrophysiol. 1999; 22(5): 695-697, indexed in Pubmed: 10353126.

27. Erkapic D, Duray GZ, Bauernfeind T, et al. Insulation defects of thin high-voltage ICD leads: an underestimated problem? J Cardiovasc Electrophysiol. 2011; 22(9): 1018-1022, doi: 10.1111/j.1540-8167.2011.02055.x, indexed in Pubmed: 21457385. Salgado R, Martín J, Martínez J, et al. Small-Caliber Lead Failure After Generator Exchange. J Cardiovasc Electrophysiol. 2016; 27(7): 846-850, doi: 10.1111/jce.12994, indexed in Pubmed: 27090984.

28. Mehta NK, Taylor M, Kalbfleisch S. The Impact of Inappropriate Implantable Cardiac Defibrillator Shocks on Cardiovascular Morbidity and Mortality. Pacing Clin Electrophysiol. 2016; 39(8): 858-862, doi: 10.1111/pace.12890, indexed in Pubmed: 27197050 .

29. Yang XW, Hua W, Ding LG, et al. OptiVol fluid index predicts acute decompensation of heart failure with a high rate of unexplained events. J Geriatr Cardiol. 2013; 10(3): 253-257, doi: 10.3969/j. issn.1671-5411.2013.03.012, indexed in Pubmed: 24133513. 